\title{
Advances in multiple sclerosis research in 2009
}

\author{
Stefan Nessler • Wolfgang Brück
}

Received: 25 June 2010/ Accepted: 20 July 2010/Published online: 6 August 2010

(C) The Author(s) 2010. This article is published with open access at Springerlink.com

\begin{abstract}
The following review summarizes the progress in multiple sclerosis research published in the Journal of Neurology in 2009.
\end{abstract}

Keywords Multiple sclerosis · Neuromyelitis optica . Advances

\section{Introduction}

Multiple sclerosis is a chronic demyelinating neuroinflammatory disease of the central nervous system of unknown etiology. Clinical disease usually starts with recurrent and reversible neurological deficits in early adulthood and transforms into a disease course with continuous and progressive neurological decline 20 years later. Inflammation composed of mononuclear cells, breakdown of the blood brain barrier, focal plaques of demyelination and axonal damage characterize acute MS lesions and can be effectively targeted by anti-inflammatory therapies. The progressive disease phase is characterized by a persistent low-level inflammatory response which eludes current treatment efforts.

\section{Etiology: genetic and environmental factors}

Multiple sclerosis is most likely caused by a complex interplay between polygenetic and environmental factors. Genetic susceptibility to multiple sclerosis is associated

S. Nessler · W. Brück $(\bowtie)$

Department of Neuropathology, University Medical Center Göttingen, Robert-Koch-Str. 40, 37075 Göttingen, Germany e-mail: wbrueck@med.uni-goettingen.de with a number of immunologically relevant genes, particularly with the HLA DR15 haplotype in Caucasians. Fernandez et al. [5] studied HLA class II alleles in patients with multiple sclerosis in the Biscay province of northern Spain. They identified the HLA class II allele $D Q B 1 * 0602$ as a potentially predisposing allele in their study population. The haplotype $D R B 1 * 0101$ had a significantly lower frequency in the MS group.

The inflammatory infiltrate of MS consists mainly of mononuclear cells which are located around small veins, but which also can infiltrate white and to a lesser extent grey matter. Migration of inflammatory cells from the blood stream to the CNS requires the interaction of adhesion molecules, including the family of selectins. Fenoglio et al. [4] studied single nucleotide polymorphisms of the selectin gene cluster in MS patients and controls of Caucasian origin, but no influence on disease susceptibility was observed.

Environmental factors currently implicated in the pathogenesis of MS include viral infections, vitamin D deficiency and smoking. Vitamin D, which is converted in its active form by sunlight exposure to the skin, is an immunemodulator and vitamin $\mathrm{D}$ supplementation has a protective effect. Current knowledge about hypovitaminosis D as an environmental risk factor for MS has been thoroughly summarized by Pierrot-Deseilligny [16]. Although sunlight exposure and vitamin D levels are diminished in a significant number of the inhabitants of Alaska, Wallin et al. [22] found a low MS prevalence in this area.

\section{Clinical presentation}

Many patients who later develop MS present first with a clinically isolated syndrome, which is usually categorized 
in terms of topography. The presence of facial sensory symptoms was associated with a decreased risk for the development of clinically definite MS. Clinical predictors of early second demyelinating events were identified in a study from the UCSF MS Center [15]. These included younger age at presentation, non-white ethnicity and a lower number of affected functional systems. Analysing data obtained in the national German MS Registry, Stuke [18] report sensory problems, paresis and visual dysfunction as the most frequently reported initial MS symptoms. Fatigue, bladder dysfunction, spasticity and ataxia became eminent with longer disease duration.

Using a questionnaire approach in 18 different European countries, Messmer [13] describes the factors which influence the employment status of people with multiple sclerosis. Symptoms associated with a negative impact on employment were mobility problems, visual impairment and memory problems. Fatigue, previously described as important for the decision to leave work, did not reach significance in this study. Financial aspects and workplace parameters such as accessibility and flexible work schedules were significant factors for staying employed.

The influence of the lifestyle factor smoking on disease progression in multiple sclerosis was studied in a prospective population based study in Tasmania [17]. Smoking was positively associated with clinical disability progression over a 3-year observation period.

Natural history studies of MS previously revealed many similarities between patients suffering from primary progressive disease (PPMS) and patients who reached a secondary progressive disease course (SPMS) after an initial relapsing remitting one. Comparing PPMS with SPMS in a Canadian MS study population, Tremlett et al. [20] report that SPMS patients enter the progressive disease phase at a later age than primary progressive patients.

\section{Paraclinical parameters}

The diagnosis of MS requires the dissemination of the disease in time and space. This can be documented on clinical grounds or by paraclinical and laboratory parameters. Magnetic resonance imaging (MRI), cerebrospinal fluid (CSF) and electrophysiological studies contribute considerably to an early and definitive MS diagnosis.

Oligoclonal band-negative and -positive patients were studied by Huttner et al. [8] with respect to the fulfilment of Barkhof's diagnostic MRI criteria for MS. The two patient groups did not differ in overall fulfilment of these criteria, but OCB-negative patients had significantly more juxtacortical lesions and fewer infratentorial lesions than OCB-positive patients.
Applying quantitative MRI measurements such as magnetization transfer ratio (MTR) to a group of secondary progressive MS patients, significant correlations were obtained between multiple sclerosis functional composite scores (MSFC) and MTR values in normal appearing white matter (NAWM), lesions and grey matter. Grey matter MTR values emerged as the best predictors of MSFC scores [6]. Significant correlations between MRI MTR measures and EDSS were not found. These, however, could be obtained for transcranial magnetic stimulation (TMS) measurements and EDSS in a study by Conte et al. [3].

Extensive studies with CSF and serum samples of MS patients and controls have not established a specific and sensitive diagnostic biomarker for this disease. In this respect, Massa et al. [11] also failed to establish the serum urate concentration as a strong predictor of MS risk in a prospective study.

\section{Neuromyelitis optica}

Neuromyelitis optica (NMO) was once considered to be an uncommon variant of MS, but is regarded now as a disease in its own right. In a population-based study in the multiethnic population of Cuba, NMO prevalence was 0.52/ 100000 with no difference by ethnicity [2]. Antibodies against the water channel protein aquaporin-4 (AQP-4) are relatively specific for this disease and useful to distinguish NMO from MS. Interleukin-6, which is elevated in the CSF of NMO patients but not in the CSF of MS patients, might be another potential biomarker to differentiate these demyelinating disorders [21]. Atypical or limited clinical variants of neuromyelitis optica have been defined by the presence of AQP-4 antibodies in a number of patients presenting with inflammatory CNS disease in a retrospective study in Tuscany [1].

\section{Therapy}

The current MS therapy is based on immunomodulatory drugs such as interferon-beta and glatiramer acetate, immunosuppressive therapies such as mitoxantrone and biologics such as natalizumab, which target specific molecules on the cell surface of immune cells. Treatment involves also the management of MS symptoms.

Drugs work only if patients take them, and patient adherence to therapy is a relevant clinical problem in many chronic diseases. Treatment adherence to injected diseasemodifying therapies in MS was investigated in a multicenter observational study by Treadaway et al. [19], which reported that the non-adherence rate was in the range of 
36-39\%. The most often reported reason for missing injections was that patients simply forgot to administer them. Older age at diagnosis and no prior disease-modifying therapy favoured adherence.

Hutchinson et al. [7] studied the efficacy of natalizumab therapy in subgroups of AFFIRM and SENTINEL which included prior treatment-naive MS patients with highly active relapsing MS (AFFIRM) or highly active disease despite interferon- $\beta$ treatment (SENTINEL). Highly active MS was defined as $\geq 2$ relapses/year prior to study entry and $\geq 1 \mathrm{Gd}+$ lesion on $\mathrm{T} 1$ weighted MRI at study entry. Natalizumab reduced the annualized relapse rate by $81 \%$ in the AFFIRM and $76 \%$ in the SENTINEL trial and the risk of disability progression by $64 \%$ in the former and $58 \%$ in the latter study, demonstrating efficacy also in highly active disease.

Serious adverse events in MS therapy have become increasingly eminent with approved monoclonal antibody therapies for highly active relapsing remitting disease. Johnson et al. [9] analyzed the willingness of MS patients to accept potential life-threatening adverse events in exchange for therapeutic benefits. They found that MS patients were willing to accept serious adverse events (SAE), such as death or disability from progressive multifocal leukoencephalopathy, at an annual risk of $0.38 \%$ in return for a decrease in relapse rate and disease progression. In fact, the mean maximum acceptable annual risk of SAE patients are willing to accept is higher than the observed risk for currently marketed therapies.

Teriflunomide is one of a number of oral MS therapeutics being tested in phase III clinical trials at the moment. Merrill et al. [12] tested the efficacy of this anti-inflammatory drug, which targets a mitochondrial enzyme important for the de novo pyrimidine synthesis, in a chronic relapsing animal model for MS. Teriflunomide administered to DA rats at disease onset or at the onset of remission prompted a more rapid recovery or reduced the severity of a clinical relapse, respectively. Clinical improvements were accompanied by fewer inflammatory infiltrates and less demyelination and axonal damage in histopathology.

Neuropsychological deficits in MS include impairments in both processing speed and memory and affect 40-60\% of patients. In a placebo-controlled double-blind trial by Morrow et al. [14], patients with cognitive dysfunctions were randomized to L-amphetamine sulfate or placebo at a 2:1 ratio. No significant differences were observed in the subjective rating of cognition or in measured processing speed, which were the primary functions tested in the study. However, cognitively impaired MS patients on L-amphetamine significantly improved on auditory/verbal and visual/spatial memory tests.

Fatigue is one of the most frequent symptoms in patients with MS and a considerable number of MS patients consider fatigue to be their most serious symptom. Treatment options include various medications such as modafinil, as well as non-pharmacological interventions. The effect of modafinil treatment on fatigue was studied by Lange et al. [10] in a double-blind placebo-controlled study. Modafinil treatment reduced fatigue, improved focused attention and also enhanced fine motoric performances and cognitive tasks in their study.

\section{Summary and outlook}

Although the etiology of multiple sclerosis remains elusive, substantial progress has been made in the treatment of the inflammatory component of this disease. Upcoming new therapies, which include oral medications, will further broaden the therapeutic repertoire and will emphasize the importance of weighing treatment benefits against potential risks.

Open Access This article is distributed under the terms of the Creative Commons Attribution Noncommercial License which permits any noncommercial use, distribution, and reproduction in any medium, provided the original author(s) and source are credited.

\section{References}

1. Bizzoco E, Lolli F, Repice AM, Hakiki B, Falcini M, Barilaro A, Taiuti R, Siracusa G, Amato MP, Biagioli T, Lori S, Moretti M, Vinattieri A, Nencini P, Massacesi L, Mata S (2009) Prevalence of neuromyelitis optica spectrum disorder and phenotype distribution. J Neurol 256:1891-1898

2. Cabrera-Gomez JA, Kurtzke JF, Gonzalez-Quevedo A, LaraRodriguez R (2009) An epidemiological study of neuromyelitis optica in Cuba. J Neurol 256:35-44

3. Conte A, Lenzi D, Frasca V, Gilio F, Giacomelli E, Gabriele M, Bettolo CM, Iacovelli E, Pantano P, Pozzilli C, Inghilleri M (2009) Intracortical excitability in patients with relapsingremitting and secondary progressive multiple sclerosis. J Neurol 256:933-938

4. Fenoglio C, Scalabrini D, Piccio L, De RM, Venturelli E, Cortini F, Villa C, Serpente M, Parks B, Rinker J, Cross AH, Bresolin N, Scarpini E, Galimberti D (2009) Candidate gene analysis of selectin cluster in patients with multiple sclerosis. J Neurol 256:832-833

5. Fernandez O, Antiguedad A, Pinto-Medel MJ, Mendibe MM, Acosta N, Oliver B, Guerrero M, Papais-Alvarenga M, Fernandez-Sanchez V, Leyva L (2009) HLA class II alleles in patients with multiple sclerosis in the Biscay province (Basque country, Spain). J Neurol 256:1977-1988

6. Hayton T, Furby J, Smith KJ, Altmann DR, Brenner R, Chataway J, Hughes RA, Hunter K, Tozer DJ, Miller DH, Kapoor R (2009) Grey matter magnetization transfer ratio independently correlates with neurological deficit in secondary progressive multiple sclerosis. J Neurol 256:427-435

7. Hutchinson M, Kappos L, Calabresi PA, Confavreux C, Giovannoni G, Galetta SL, Havrdova E, Lublin FD, Miller DH, O'Connor PW, Phillips JT, Polman CH, Radue EW, Rudick RA, Stuart WH, Wajgt A, Weinstock-Guttman B, Wynn DR, Lynn F, 
Panzara MA (2009) The efficacy of natalizumab in patients with relapsing multiple sclerosis: subgroup analyses of AFFIRM and SENTINEL. J Neurol 256:405-415

8. Huttner HB, Schellinger PD, Struffert T, Richter G, Engelhorn T, Bassemir T, Maurer M, Garcia M, Schwab S, Kohrmann M, Doerfler A (2009) MRI criteria in MS patients with negative and positive oligoclonal bands: equal fulfillment of Barkhof's criteria but different lesion patterns. J Neurol 256:1121-1125

9. Johnson FR, Van HG, Ozdemir S, Hass S, White J, Francis G, Miller DW, Phillips JT (2009) Multiple sclerosis patients' benefit-risk preferences: serious adverse event risks versus treatment efficacy. J Neurol 256:554-562

10. Lange R, Volkmer M, Heesen C, Liepert J (2009) Modafinil effects in multiple sclerosis patients with fatigue. J Neurol 256:645-650

11. Massa J, O'Reilly E, Munger KL, Delorenze GN, Ascherio A (2009) Serum uric acid and risk of multiple sclerosis. J Neurol 256:1643-1648

12. Merrill JE, Hanak S, Pu SF, Liang J, Dang C, Iglesias-Bregna D, Harvey B, Zhu B, McMonagle-Strucko K (2009) Teriflunomide reduces behavioral, electrophysiological, and histopathological deficits in the Dark Agouti rat model of experimental autoimmune encephalomyelitis. J Neurol 256:89-103

13. Messmer UM, Specchia C, Battaglia MA, Miller DM (2009) Factors that influence the employment status of people with multiple sclerosis: a multi-national study. J Neurol 256: 1989-1996

14. Morrow SA, Kaushik T, Zarevics P, Erlanger D, Bear MF, Munschauer FE, Benedict RH (2009) The effects of L-amphetamine sulfate on cognition in MS patients: results of a randomized controlled trial. J Neurol 256:1095-1102
15. Mowry EM, Pesic M, Grimes B, Deen SR, Bacchetti P, Waubant E (2009) Clinical predictors of early second event in patients with clinically isolated syndrome. J Neurol 256:1061-1066

16. Pierrot-Deseilligny C (2009) Clinical implications of a possible role of vitamin D in multiple sclerosis. J Neurol 256:1468-1479

17. Pittas F, Ponsonby AL, van der Mei IA, Taylor BV, Blizzard L, Groom P, Ukoumunne OC, Dwyer T (2009) Smoking is associated with progressive disease course and increased progression in clinical disability in a prospective cohort of people with multiple sclerosis. J Neurol 256:577-585

18. Stuke K, Flachenecker P, Zettl UK, Elias WG, Freidel M, Haas J, Pitschnau-Michel D, Schimrigk S, Rieckmann P (2009) Symptomatology of MS: results from the German MS Registry. J Neurol 256:1932-1935

19. Treadaway K, Cutter G, Salter A, Lynch S, Simsarian J, Corboy J, Jeffery D, Cohen B, Mankowski K, Guarnaccia J, Schaeffer L, Kanter R, Brandes D, Kaufman C, Duncan D, Marder E, Allen A, Harney J, Cooper J, Woo D, Stuve O, Racke M, Frohman EM (2009) Factors that influence adherence with disease-modifying therapy in MS. J Neurol 256:568-576

20. Tremlett H, Zhao Y, Devonshire V (2009) Natural history comparisons of primary and secondary progressive multiple sclerosis reveals differences and similarities. J Neurol 256:374-381

21. Uzawa A, Mori M, Ito M, Uchida T, Hayakawa S, Masuda S, Kuwabara S (2009) Markedly increased CSF interleukin-6 levels in neuromyelitis optica, but not in multiple sclerosis. J Neurol 256:2082-2084

22. Wallin MT, Page WF, Kurtzke JF (2009) Migration and multiple sclerosis in Alaskan military veterans. J Neurol 256:1413-1417 